\title{
Optimal Design of Cellular Material Systems for Crashworthiness
}

\author{
Kai Liu ${ }^{1}$, ZongYing $\mathrm{Xu}^{2}$, Duane Detwiler ${ }^{3}$, Andres Tovar ${ }^{2}$ \\ ${ }^{1}$ Purdue University, ${ }^{2}$ Indiana University Purdue University Indianapolis, ${ }^{3}$ Honda R\&D Americas, Inc.
}

CITATION: Liu, K., Xu, Z., Detwiler, D., and Tovar, A., "Optimal Design of Cellular Material Systems for Crashworthiness," SAE Technical Paper 2016-01-1396, 2016, doi:10.4271/2016-01-1396.

\begin{abstract}
This work proposes a new method to design crashworthiness structures that made of functionally graded cellular (porous) material. The proposed method consists of three stages: The first stage is to generate a conceptual design using a topology optimization algorithm so that a variable density is distributed within the structure minimizing its compliance. The second stage is to cluster the variable density using a machine-learning algorithm to reduce the dimension of the design space. The third stage is to maximize structural crashworthiness indicators (e.g., internal energy absorption) and minimize mass using a metamodel-based multi-objective genetic algorithm. The final structure is synthesized by optimally selecting cellular material phases from a predefined material library. In this work, the Hashin-Shtrikman bounds are derived for the two-phase cellular material, and the structure performances are compared to the optimized structures derived by our proposed framework. In comparison to traditional structures that made of a single cellular phase, the results demonstrate the improved performance when multiple cellular phases are used.
\end{abstract}

\section{Introduction}

Design for crashworthiness - which involves the analysis, synthesis, and optimization of protective vehicle components/systems during a crash event - is a long and computationally expensive engineering task. To reduce the design cycle time and increase the effectiveness of the structural component for crashworthiness, researchers have integrated design optimization methods [1], [2]. However, such integration is hindered by two technical challenges: conceptual design generation and non-linearity of the structural component. The first challenge, conceptual design generation, refers to the conception of the type of crush initiator, cellular pattern, or foam density distribution. This design is generally unknown and the designer has to test several configurations before committing to a design that can be further parameterized and optimized. The second challenge, nonlinearity of the crash computational model, imposes a great computational cost on the simulation, which makes it impractical to use traditional optimization methods.

To systematically address the conceptual design generation of crashworthy structures, researchers have explored the use of topology optimization methods (material distribution) using analytical approximations of the sensitivity coefficients [3]. Methods based on linear implicit finite element analysis, such as equivalent static loads (ESL) [4], [5], or partially non-linear implicit methods [6], [7] are numerically efficient sicne sensitivity coefficients can be obtained; however, these methods are not applicable to capture all the relevant aspects of the transient crash event. Truly non-linear explicit methods have been applied through heuristic methods, leading to innovative and useful conceptual designs [8]-[14]. Some of the main developments have Page 1 of 8 been achieved by the hybrid cellular automaton (HCA) method proposed by the corresponding author and collaborators [12]-[14]. The premise of the method is that high-energy absorbing structures can be synthesized by uniformly distributing the internal energy density in a voxel-based discretized design domain.

To reduce the computational cost of crashworthiness design problems, metamodel methodologies have been frequently employed to replace the actual simulation models [15]-[18]. However, the performances of metamodel may vary from problem to problem, researchers have comparatively studied different metamodels for the use in crashworthiness design [19]-[21]. As a result of advances in computer throughput, multiple sophisticated, expensive metamodels and multi-objective have been incorporated into structure crashworthiness design [22]-[28]. Recently, cellular materials, e.g., foams, sawdust, honeycomb, have been increasingly used in crashworthiness applications. Theses (functionally-graded) cellular structures are filled in straight [29]-[32] and tapered [33]-[35] thinwalled structures in order to increase the crashworthiness without sacrificing too much on weight. At the material level, numerous studies in the literature discussed the design of composite materials using optimization method to improve energy absorption capabilities [36]-[40]. However, the design algorithms for crashworthy structure and its cellular material can be barely found in the literature.

The objective of this work is to propose a new method to design cellular system for crashworthiness. In our previous work, we proposed a systemic approach to design and optimize thin-walled tubular component [13], [14], [41]. The energy absorbing capabilities of the thin-walled components depend on the plastically deformable progressive crushing zones. The progressive crushing zones were generated using the principle of compliant mechanism. With an efficient initial design, we used shell thickness as design variables and applied our proposed three-stage design optimization algorithm to design crashworthiness structures [21]. In this investigation, the three-stage design optimization algorithm is revised for designing cellular material system with crashworthiness consideration. The conceptual design is generated using topology optimization to maximize structure stiffness. Unsupervised machine learning - Kmeans - is applied to characterize topology optimization results. With a limited number of design variables, we are able to search over the entire design space to find the best cellular material for each cluster within a given material library. The results demonstrate remarkable improvements in all crashworthiness measures that are considered in this study as compared to the cellular materials that found on the Hashin-Shtrikman upper bound.

The rest of the paper is organized in three main sections: conceptual design generation, design parameterization, and design optimization. The summary and discussion of the results are presented in the final section. 


\section{Conceptual Design}

\section{Problem definition}

To generalize a conceptual design, structural optimization approach is used. If a material property is characterized by a parameter $x \in \mathbb{R}$, where $0 \leq x \leq 1$, then the conceptual design problem is to find the distribution of all possible parameterized materials $\mathbf{x} \in \mathbb{R}^{n}$ in a discretized structure with $n$ discrete elements that maximize an objective function $f(\mathbf{x})$ subject to equality $\mathbf{h}(\mathbf{x})$ and inequality $\mathbf{g}(\mathbf{x})$ constraints. In this investigation, traditional topology optimization problem is solved with only mass constraint. The design problem is shown in Figure 1. A structure is subject to a $0.0022 \mathrm{~kg}$ rigid pole impact, where the pole is prescribed with $10 \times 10^{3} \mathrm{~mm} / \mathrm{s}$ velocity. The rectangular domain is $800 \mathrm{~mm} \times 100 \mathrm{~mm} \times 80 \mathrm{~mm}$. Only half of the structure is considered as a design domain due to symmetricity of the problem. The structure is discretized using $5 \mathrm{~mm} \times 5 \mathrm{~mm} \times 5 \mathrm{~mm}$ brick elements. The problem parameters are summarized in Table 1.

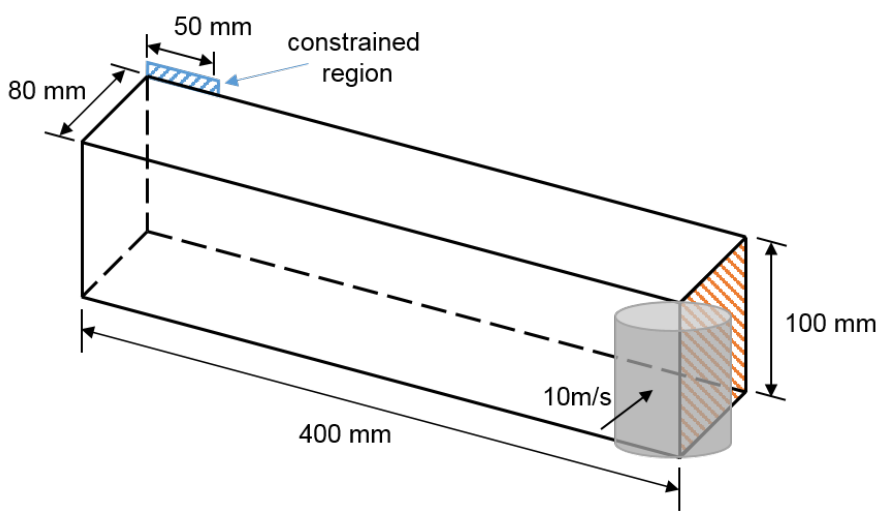

Figure 1 Design domain for the beam design problem.

Mathematically, the topology optimization problem is the following:

$$
\begin{aligned}
\text { find } & \mathbf{x} \in \mathbb{R}^{n} \\
\text { minimize } & f(\mathbf{x})=\mathbf{F}^{\mathrm{T}} \mathbf{U}(\mathbf{x}) \\
\text { subject to } & m(\mathbf{x})-\bar{m} \leq 0 \\
& \mathbf{0} \leq \mathbf{x} \leq \mathbf{1}
\end{aligned}
$$

where the equilibrium conditions $\mathbf{K}(\mathbf{x}) \mathbf{U}(\mathbf{x})=\mathbf{F}$ is satisfied. $\mathbf{K}(\mathbf{x})$ is the global stiffness matrix of the structure, $\mathbf{U}(\mathbf{x})$ and $\mathbf{F}$ are the global displacement and force vector, respectively. The material model used in Eq. (1) is:

$$
E(x)=x E_{1} \text {, }
$$

where $E_{1}$ is the Elastics modulus of solid material as given in Table 2 .

Table 1 Design problem parameters

\begin{tabular}{cc}
\hline Parameter & Value \\
\hline Dimensions & $400 \times 100 \times 80 \mathrm{~mm}^{3}$ \\
FEA mesh & $160 \times 20 \times 16$ \\
Mass fraction $\bar{m}$ & 0.25 \\
\hline
\end{tabular}

Table 2 Base material properties used in this study.

\begin{tabular}{cc}
\hline Property & Value \\
\hline Density & $2.70 \times 10^{-9} \mathrm{~kg} / \mathrm{mm}^{3}$ \\
Elastic modulus & $70 \times 10^{3} \mathrm{~N} / \mathrm{mm}^{2}$ \\
Poisson's ratio & 0.33 \\
\hline
\end{tabular}

In this stage, we are assuming small strain and displacement. Since the material is also elastic, only a set of unit force is applied. After performing 35 iterations using top3d [42], the topology optimized structure in Figure 2. This final topology contains 23685 distinct design variable values.

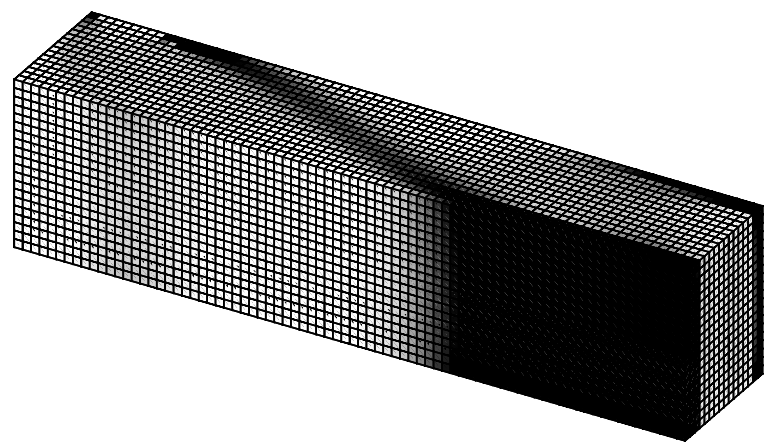

Figure 2 The final topology generated for the beam problem.

\section{Design Parameterization}

The conceptual design is generated using over ten-thousand design variables. It is impractical to utilize general optimization schemes with such a high number of design variables. In addition, the physical representation of the intermediate values $x \in(0,1)$ is unclear, even impractical. To overcome the above problems, one major task of this investigation is to reduce the dimension of design space. The reduced number of design variables also increases the manufacturability of the optimized design. In this work, we propose the use of unsupervised machine learning techniques to reduce the design space dimensionality. One promising unsupervised machine learning technique is $\mathrm{K}$-means clustering.

\section{K-means clustering}

K-means, first used by James MacQueen in 1967 [43], remains as one of the most popular unsupervised machine learning techniques. In this method, given a set of observations $\mathbf{x} \in \mathbb{R}^{n}$ the algorithm aims to partition the $n$ observations into $K$ sets $\mathbf{S}=\left\{S_{1}, \ldots, S_{K}\right\}$ where $K \leq$ $n$. The objective is to minimize the within-cluster sum of squares defined as

$$
\begin{aligned}
\text { find } & \boldsymbol{\mu} \in \mathbb{R}^{K} \\
\operatorname{minimize} & J(\mu)=\sum_{k=1}^{K} \sum_{x_{i} \in S_{k}}\left\|x_{i}-\mu_{k}\right\|^{2},
\end{aligned}
$$


Where $\mu_{k}$ is the mean of points in $S_{k}$. Commonly, an iterative refinement algorithm is used to perform K-means clustering [44]. The procedure of classifying data follows some simple steps as shown in Algorithm 1. This algorithm returns element indices for each cluster.

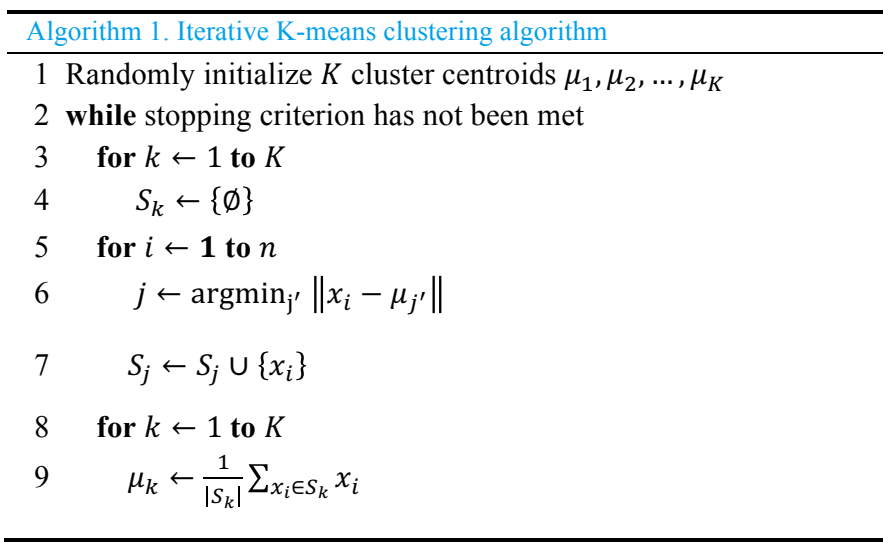

The K-means clustering result is heavily dependent on the initial cluster centers (the first step in Algorithm 1), which is neither always global optimum nor repeatable. To increase the chance of K-means algorithm converging to the global minima, multiple restarts (typically 50-1000 times) can be used and clustering that gave lowest cost Eq. (3) can be picked. However, this method is inefficient. As an alternative to the standard heuristic K-means algorithm, this investigation incorporates Ckmeans.1d.dp. Ckmeans.1d.dp is a software package written in $\mathrm{R}$ that implements a dynamic programming algorithm to perform optimal one-dimensional $\mathrm{K}$ means clustering [45]. It guarantees optimality and repeatability [46].

\section{Optimal $K$ value}

In K-means, the number $K$ represents the number of means (clusters) one wants to partition for a set of observations. The choice of optimal $K$ value is always ambiguous, especially for not well-separated data sets. To enhance manufacturability as well as effective use of surrogate models later, the optimal $K$ value in this study is selected in the range of 2 and 10.

Figure 3 shows how the objective value in Eq. (3) varies with number of clusters $K$. As can be seen from the figure, an elbow appears around $K=3$ and $K=4$. Therefore, we could choose either 3 or 4 as the optimal value for $K$. In this investigation, both 3 and 4 are studies in the design optimization stage. Figure 4 and Figure 5 show the topology optimized structure (Figure 2) been clustered into 3 and 4 groups, respectively. The contour indicates different clusters.

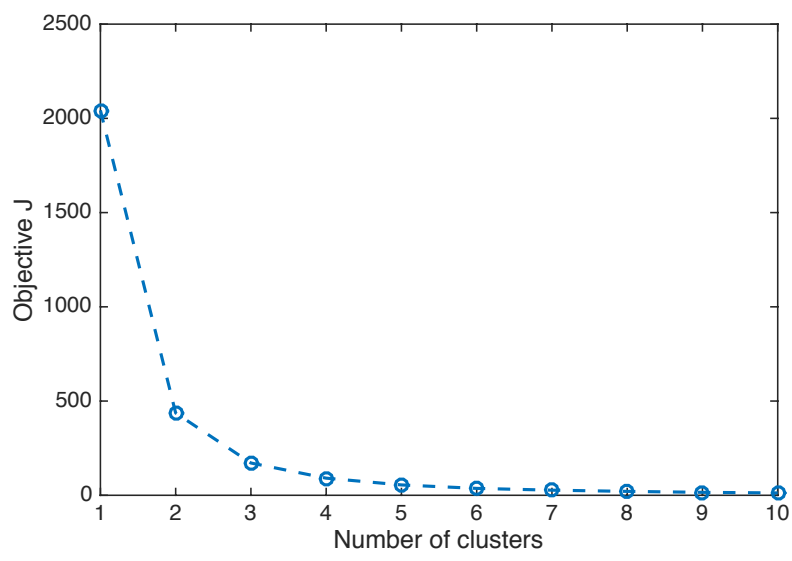

Figure 3 K-means clusters vs. Objective
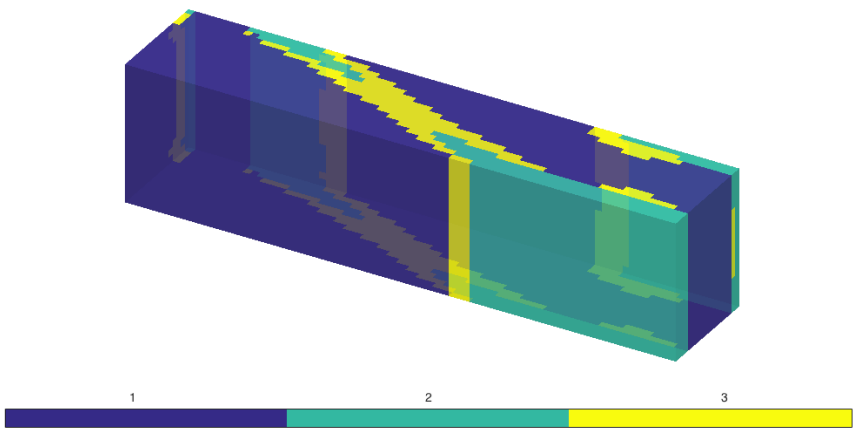

Figure 4 Clustering Figure 2 into 3 groups

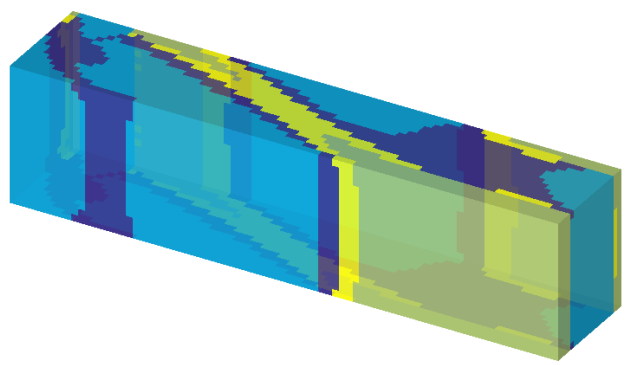

Figure 5 Clustering Figure 2 into 4 groups

\section{Design Optimization}

After design parameterization using $\mathrm{K}$-means, design space is reduced from $\mathbb{R}^{n}$ to $\mathbb{R}^{K}$. The design optimization stage is to find the best cellular material for each cluster that maximize structure internal energy absorption and minimize mass. As a first step into crashworthiness design using cellular materials, we are limited ourselves within a predefined cellular material library. 


\section{Cellular material library}

This work considers elastic isotropic materials. Four types of cellular materials are generated by varying the size of cubic hole in the center as shown in Figure 6. The material properties are characterized using homogenization method [47] and are summarized in Table 3.
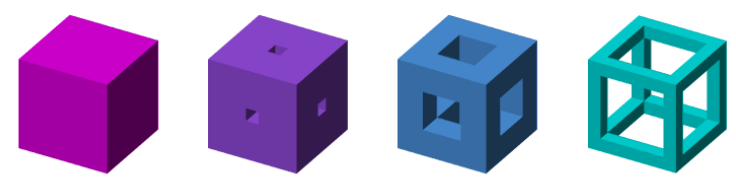

Figure 6 Cellular Material Structures.

Table 3 Cellular Material Library, ID increases from left to right in Figure 6.

\begin{tabular}{ccccc}
\hline $\begin{array}{c}\text { Material } \\
\text { ID }\end{array}$ & $\begin{array}{c}\text { Density } \\
\left(\mathrm{kg} / \mathrm{mm}^{3}\right)\end{array}$ & $\begin{array}{c}\text { Elastic modulus } \\
\left(\mathrm{N} / \mathrm{mm}^{2}\right)\end{array}$ & $\begin{array}{c}\text { Poisson's } \\
\text { ratio }\end{array}$ & $\begin{array}{c}\text { Volume } \\
\text { fraction }\end{array}$ \\
\hline 1 & $2.70 \times 10^{-9}$ & $70.00 \times 10^{3}$ & 0.33 & 1.00 \\
2 & $2.70 \times 10^{-9}$ & $62.53 \times 10^{3}$ & 0.33 & 0.94 \\
3 & $2.70 \times 10^{-9}$ & $20.01 \times 10^{3}$ & 0.36 & 0.61 \\
4 & $2.70 \times 10^{-9}$ & $13.89 \times 10^{3}$ & 0.46 & 0.20 \\
\hline
\end{tabular}

The Hashin-Shtrikman bounds for this two-phase cellular material are calculated using [48]:

$$
\begin{gathered}
K_{0}^{*}=K_{0}+\frac{v_{1}}{\frac{1}{K_{1}-\mathrm{K}_{0}}+\frac{3 v_{0}}{3 K_{0}+4 G_{0}}}, \\
K_{1}^{*}=K_{1}+\frac{v_{0}}{\frac{1}{K_{0}-\mathrm{K}_{1}}+\frac{3 v_{1}}{3 K_{1}+4 G_{1}}}, \\
G_{0}^{*}=G_{0}+\frac{v_{1}}{\frac{1}{G_{1}-\mathrm{G}_{0}}+\frac{6\left(K_{0}+2 G_{0}\right) v_{0}}{5 G_{0}\left(3 K_{0}+4 G_{0}\right)}}, \\
G_{1}^{*}=G_{1}+\frac{v_{0}}{\frac{1}{G_{0}-\mathrm{G}_{1}}+\frac{6\left(K_{1}+2 G_{1}\right) v_{1}}{5 G_{1}\left(3 K_{1}+4 G_{1}\right)}},
\end{gathered}
$$

where the subscript 0 or 1 indicate void or solid material. $v_{(\cdot)}, K_{(\cdot)}$ and $G_{(\cdot)}$ denote the Poisson's ratio, bulk moduli and shear moduli, respectively. The bounds for the Young's modulus are calculated by using the relation $E^{*}=9 K^{*} G^{*} /\left(8 K^{*}+G^{*}\right)$ and are shown in Figure 7. The four cellular material points are fitted using a polynomial function. $\mathrm{H}-\mathrm{S}$ bounds show that the materials in the library are within the bounds, and the material model we used earlier in the conceptual design generation stage does not satisfy the H-S bounds-see Eq. (2).

Page 4 of 8

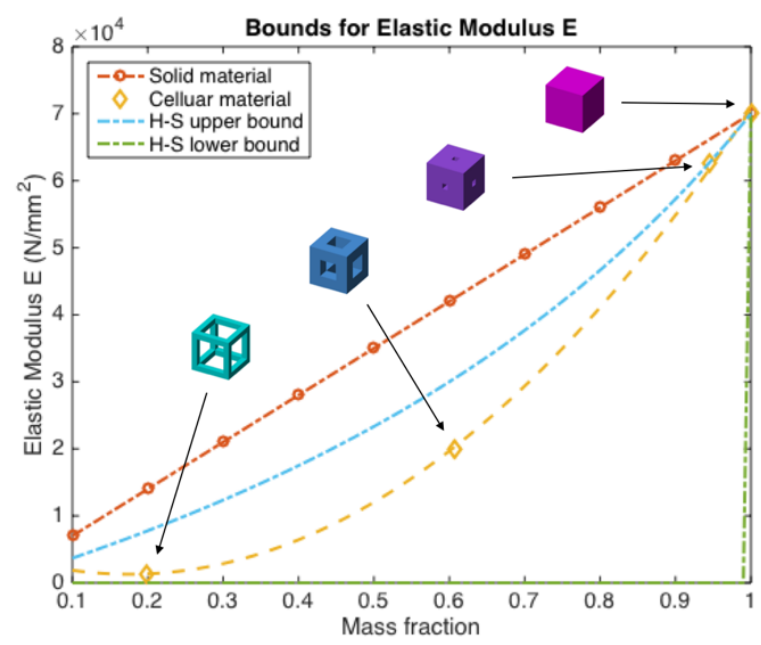

Figure 7 Hashin-Shtrikman Bounds for Elastic Modulus.

\section{Problem formulation}

To find the optimal clusters' cellular structure with maximized crashworthiness, we proposed a conflict multi-objective design optimization problem. For solid structures, the structure internal energy is proportional to structure mass, i.e., higher internal energy along with higher mass. By replacing solid parts with different cellular materials, we aim to find structures (Pareto fronts) that have high internal energy as well as low mass. Mathematically, this multiobjective optimization problem is defined as:

$$
\begin{aligned}
\text { find } & \text { MID } \in \mathbb{N}^{K} \\
\text { maximize } & \text { Internal Energy } \\
\text { minimize } & \text { Mass } \\
\text { subject to } & M I D_{k} \in\{1,2,3,4\} \\
& k=1, \ldots, K
\end{aligned},
$$

where $\mathbb{N}$ is the natural numbers that exclude $0, M I D_{k}$ is the material ID (see Table 3) for cluster $k$. This proposed multi-objective programming problem is solved by metamodeling and global optimization algorithms. Some key steps are described in the following subsections.

\section{Design of experiments}

Design of experiments is the selection procedure for finding the points in the design space that must be analyzed. Many strategies can be used to sample the design points [15], e.g., the factorial, Doptimal, and Latin hypercube designs. Since the design variables have only discretized values, D-optimal designs are used in this investigation. D-optimal designs are straights optimizations based on a chosen optimality criterion and the model that will be fit. The optimality criterion is to maximize $\left|\mathbf{X}^{\mathrm{T}} \mathbf{X}\right|$, the determinant of the information matrix $\mathbf{X}^{\mathrm{T}} \mathbf{X}$. This optimality criterion results in minimizing the generalized variance of the parameter estimates for a pre-specified model. As a result, the 'optimality' of a given Doptimal design is model dependent. The model selected in this investigation is linear model with interaction terms. That is, suppose the number of clusters $K=3$ (three design variables), then the threefactor, seven-term interaction model is: 


$$
\begin{gathered}
y=\beta_{0}+\beta_{1} X_{1}+\beta_{2} X_{2}+\beta_{3} X_{3}+\beta_{12} X_{1} X_{2}+\beta_{13} X_{1} X_{3}+\beta_{23} X_{2} X_{3} \\
+\varepsilon
\end{gathered}
$$

The levels being considered are:

$$
\begin{aligned}
& X_{1}: 4 \text { levels }(1,2,3,4) \\
& X_{2}: 4 \text { levels }(1,2,3,4) \\
& X_{3}: 4 \text { levels }(1,2,3,4)
\end{aligned}
$$

The candidate set is a full factorial in all factors containing $4^{3}=64$ possible design runs. The number of design runs in D-optimal designs used in this investigation is $10 \times K$, i.e., 30 in this example.

\section{Metamodeling}

Unlike initial conceptual design stage, this stage solves crash simulations using explicit geometry nonlinear finite element analysis (LS-Dyna). To reduce the computational cost of crashworthiness design problems, metamodel methodologies are used. Based on our previous study, Kriging is chosen to build metamodel for internal energy [21]. One challenge in this investigation is to build an accurate metamodel. This investigation uses Efficient Global Optimization (EGO) scheme to build our metamodel [49]. The EGO algorithm works as follows: after we fit an initial metamodel with $10 \times K$ samples, the expected improvement is maximized using global optimization algorithms, such as genetic algorithm, branch-andbound algorithm. If the expected improvement is less than $0.1 \%$ of the present best function value two times in a row, we stop. Otherwise, we sample the function where expected improvement is maximized, and rebuild the metamodel, and iterate. The expected improvement is defined:

$$
\mathrm{E}[I(\mathbf{x})]=\left(y_{p b s}-\hat{y}\right) \Phi\left(\frac{y_{p b s}-\hat{y}}{s}\right)+s \phi\left(\frac{y_{p b s}-\hat{y}}{s}\right),
$$

where $y_{p b s}$ is the present best function value, $\hat{y}$ is the predicted function value, $s$ is the root-mean-square error of the predictor, $\phi(\cdot)$ and $\Phi(\cdot)$ are the probability density function (PDF) and cumulative density function (CDF) of a normal distribution, respectively.

\section{Optimization}

The design optimization problem is to find the material model for each cluster within a given material library. The cluster size considered in this investigation is up to 4 clusters. Therefore, the maximum design space is $4^{4}=256$. Once the metamodel is built, the optimal can be found efficiently by using exhaustive search. One should note that the dimension of the design space growth exponentially with the number of clusters, once the computational cost of exhaustive search is no longer manageable, a more efficient global optimal search algorithm should be used.

\section{Results}

The optimization results for 3 and 4 clusters are shown in Figure 8 and Figure 9. In the figures, the entire design space is in plotted in "+", i.e., $4^{3}=64$ with $K=3$ and $4^{4}=256$ with $K=4$. "Solid material" shows the near linear relationship between structure mass and internal energy by using the linear material model Eq. although there is no physical meaning for those material except at mass fraction 0 and 1. "K1 cellular material" indicates that the whole structure is replacement by one single cellular material, total of 4 points in diamond are shown in the figure, and the dashed line is a polynomial interpolation of these 4 points. "H-S upper bound" and "H-S lower bound" are the simulation results of solid structure with material elastic modulus sampled from Figure 7.

As can be seen from Figure 8 and Figure 9, "K1 cellular material" sits between "H-S lower bound" and "H-S upper bound", which is as expected. "H-S upper bound" dominates "K1 cellular material", which indicates that our predefined cellular materials are not optimal. On the other hand, plenty of "+" points dominate the H-S upper bound, which demonstrates that the use of multiple cellular materials is more efficient than single phase cellular material.

Another notable observation from Figure 8 and Figure 9 is that the "solid material" (solid-void topologies) dominates all design experiments since the model uses artificial material properties which do not exist in the real world as illustrated in Figure 7. Additional reasons why solid-void topologies are still the optimal include: (1) our predefined cellular materials do not consider void structure, since void material will bring structure discontinuity during the simulation; (2) the material library is predefined and not necessarily optimal.

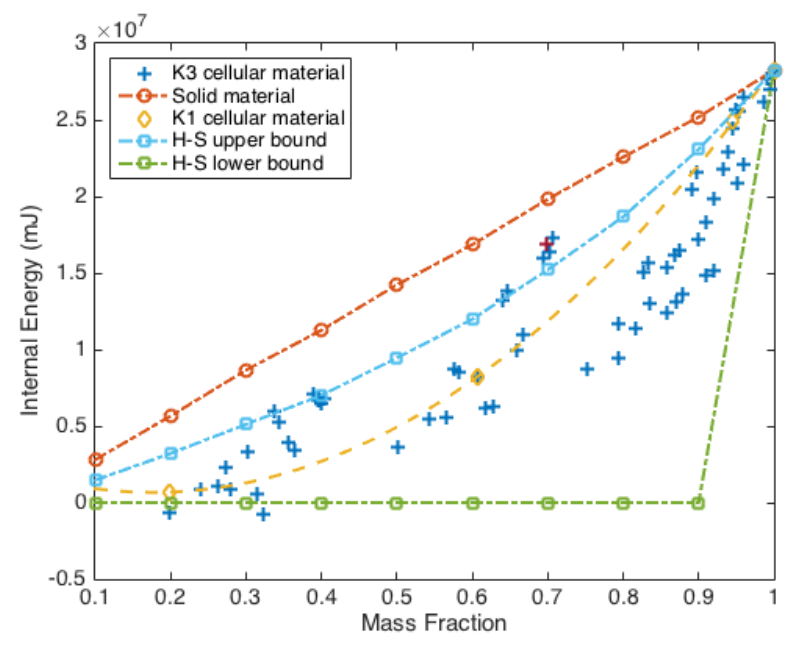

Figure 8 Design optimization results with $K=3$.

Page 5 of 8 


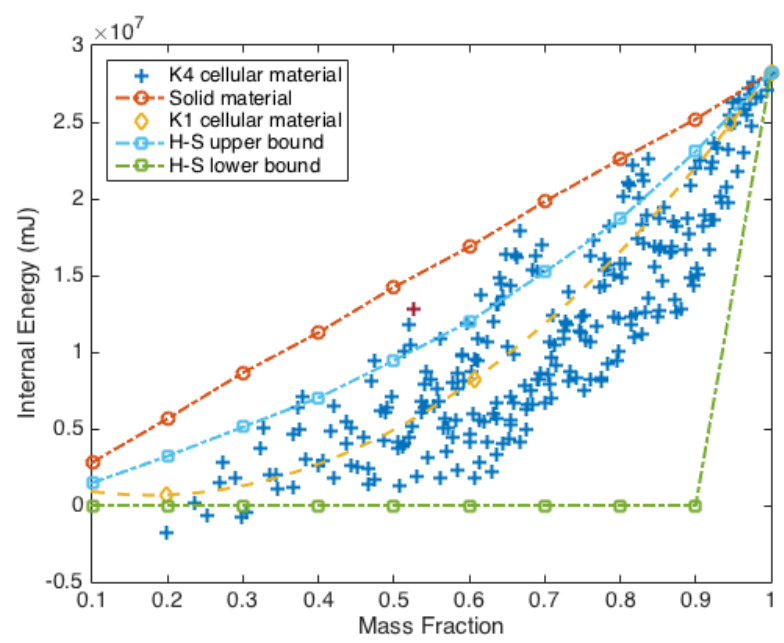

Figure 9 Design optimization results with $K=4$.

Figure 10 and Figure 11 show the Pareto-optimal solution (marked with magenta "+" in Figure 8 and Figure 9), i.e., the point closes to the utopia point. The contour represents the corresponding cellular material structure as in Figure 6. The Pareto-optimal solution for $K=$ 3 is at $\left(0.6989,1.6910 \times 10^{7}\right)$ while for $K=4$ it is located at $\left(0.5265,1.2780 \times 10^{7}\right)$. As can be seen from Figure 11, even though we have predefined 4 clusters, the Pareto-optimal solution contains only 3 different cellular materials. By using more clusters, one is able to achieve more optimal solutions that are beyond " $\mathrm{H}-\mathrm{S}$ upper bound"; by using less clusters, the optimized structures are easy to be manufactured. A good choice of number of clusters is the trade-off between performance and manufacturability.

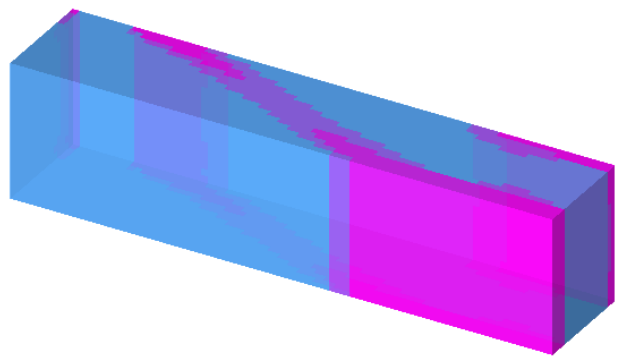

Figure 10 Pareto-optimal solution of $K=3$.

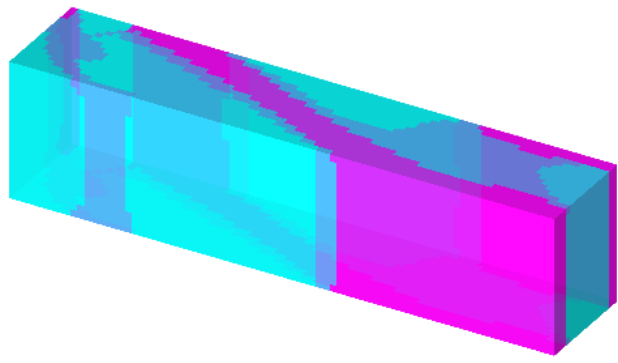

Figure 11 Pareto-optimal solution of $K=4$.

\section{Summary and Discussion}

This work proposed a method to optimally design crashworthy structures made of functionally graded cellular material. The method consists of three stages: conceptual design generation, design parameterization (clustering), and metamodel-based multi-objective design optimization. The first stage is to generate a good conceptual design using topology optimization. The result is a conceptual density distribution over the design domain. The second stage is to cluster the densities and reduce the dimension of the design space. In this work, we use an unsupervised machine learning algorithm-K-means clustering. The last stage is the design optimization of the cluster cellular structure. In this stage, global optimization algorithm is applied on the clustered structure and geometric nonlinearity is considered in the finite element analysis model. Due to the nonlinearity and complexity of the model, metamodel is used. As a first attempt to crashworthiness design using cellular materials, we mainly focused on a set of predefined cellular materials. The result is a set of Pareto optimization points that maximizes internal energy and minimizes structure mass.

In terms of internal energy absorption and mass, solid-void topologies are still dominant [42], but this work shows that designs with multiple cellular materials perform better than the ones with a single cellular material. The proposed method has proven effective on solid-shell thin-wall tubular structures subject to frontal impact [21]. This work demonstrates tremendous opportunities to advance this method and optimally design cellular vehicle structures subjected to side impact.

The current ongoing research, which includes avoiding predefined cellular materials in the third stage of the design algorithm that followed by one additional design cellular materials stage, utilizes inverse homogenization and topology optimization to design optimal cellular material for each cluster based on the results from design parameterization stage.

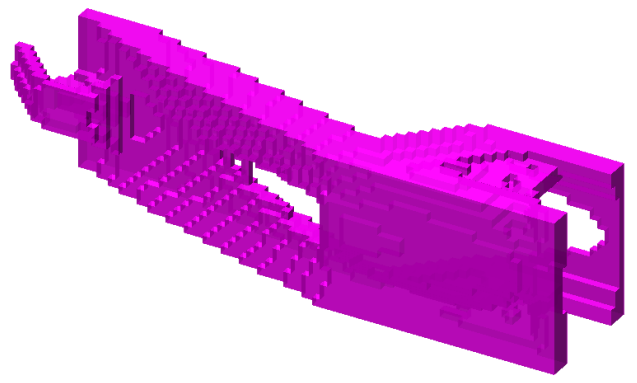

Figure 12 Topology optimized structure with mass fraction 0.25 and internal energy $8.5041 \times 10^{6} \mathrm{~mJ}$.

\section{Acknowledgments}

Honda R\&D Americas supported this research effort. Any opinions, findings, conclusions, and recommendations expressed in this investigation are those of the writers and do not necessarily reflect the views of the sponsors.

\section{References}

[1] G. Sun, G. Li, S. Hou, S. Zhou, W. Li, and Q. Li, "Crashworthiness design for functionally graded foam-filled thin- 
walled structures," Mater. Sci. Eng. A, Struct. Mater., Prop. Microstruct. Process., vol. 527, no. 7-8, pp. 1911 - 19, 2010.

[2] H. Yin, G. Wen, S. Hou, and Q. Qing, "Multiobjective crashworthiness optimization of functionally lateral graded foamfilled tubes," Mater. Des., vol. 44, no. 0, pp. 414-428, 2013.

[3] M. P. Bendsøe and O. Sigmund, Topology optimization: theory, method and applications. Springer, 2003.

[4] K. Byung-Soo, G.-J. Park, and J. S. Arora, "A review of optimization of structures subjected to transient loads," Struct. Multidiscip. Optim., vol. 31, pp. 81-95, 2006.

[5] J. K. Paik, B. J. Kim, D. K. Park, and B. S. Jang, "On quasi-static crushing of thin-walled steel structures in cold temperature: Experimental and numerical studies," Int. J. Impact Eng., vol. 38, no. 1, pp. 13-28, Jan. 2011.

[6] R. R. Mayer, N. Kikuchi, and R. A. Scott, "Application of Topological Optimization Techniques to Structural Crashworthiness," Int. J. Numer. Methods Eng., vol. 39, no. 8, pp. 1383-1403, 1996

[7] D. Jung and H. C. Gea, "Topology optimization of nonlinear structures," Finite Elem. Anal. Des. , vol. 40, no. 11, pp. 1417-1427, 2004

[8] C. A. Soto, "Structural topology optimization for crashworthiness," Int. J. Crashworthiness, vol. 9, no. 3, pp. 277-283, 2004.

[9] H. Wang, Z.-D. Ma, N. Kikuchi, C. Pierre, and B. Raju, "Multi-domain multi-step topology optimization for vehicle structure crashworthiness design," in SAE Technical Paper 2004-01-1173, 2004.

[10] P. J. Rousseeuw, "Silhouettes: a graphical aid to the interpretation and validation of cluster analysis," J. Comput. Appl. Math. , vol. 20, pp. 53-65, 1987.

[11] C. Ortmann and A. Schumacher, "Graph and heuristic based topology optimization of crash loaded structures," Struct. Multidiscip. Optim., vol. 47, no. 6, pp. 839-854, Jan. 2013.

[12] N. M. Patel, B.-S. Kang, J. E. Renaud, and A. Tovar, "Crashworthiness Design Using Topology Optimization," J. Mech. Des. , vol. 131, no. 6, p. 61013, 2009.

[13] C. Mozumder, J. E. Renaud, and A. Tovar, "Topometry optimisation for crashworthiness design using hybrid cellular automata," Int. J. Veh. Des. , vol. 60, no. 1/2, p. 100, 2012.

[14] P. Bandi, J. P. Schmiedeler, and A. Tovar, "Design of Crashworthy Structures With Controlled Energy Absorption in the Hybrid Cellular Automaton Framework," J. Mech. Des. , vol. 135, no. 9, p. 91002, 2013.

[15] R. H. Myers and D. C. M. C. M. Anderson-Cook, Response Surface Methodology: Process and Product Optimization Using Designed Experiments, 3rd ed. Wiley, 2009.

[16] H. M. Gutmann, "A radial basis function method for global optimization," J. Glob. Optim., vol. 19, no. 3, pp. 201-227, 2001.

Page 7 of 8
[17] R. G. Regis and C. A. Shoemaker, "Constrained global optimization of expensive black box Functions using radial basis functions," J. Glob. Optim., vol. 31, no. 1, pp. 153-171, 2005.

[18] J. Sacks, W. J. Welch, T. J. Mitchell, and H. P. Wynn, "Design and analysis of computer experiments," Stat. Sci., vol. 4, no. 4, pp. 409-423, 1989.

[19] N. Stander, W. Roux, M. Giger, M. Redhe, N. Fedorova, and J. Haarhoff, "A Comparison of Metamodeling Techniques for Crashworthiness Optimization," in 10th AIAA/ISSMO Multidisciplinary Analysis and Optimization Conference, 2004.

[20] H. Fang, M. Rais-Rohani, Z. Liu, and M. F. F. Horstemeyer, "A comparative study of metamodeling methods for multiobjective crashworthiness optimization," Comput. Struct., vol. 83, no. 25-26, pp. 2121-2136, Sep. 2005.

[21] K. Liu, A. Tovar, E. Nutwell, and D. Detwiler, "ThinWalled Compliant Mechanism Component Design Assisted by Machine Learning and Multiple Surrogates," in SAE Technical Paper 2015-01-1369, 2015.

[22] X. Wang and L. Shi, "A new metamodel method using Gaussian process based bias function for vehicle crashworthiness design," Int. J. Crashworthiness, vol. 19, no. 3, pp. 311-321, Mar. 2014.

[23] J. Marzbanrad and M. R. Ebrahimi, "Multi-Objective Optimization of aluminum hollow tubes for vehicle crash energy absorption using a genetic algorithm and neural networks," ThinWalled Struct., vol. 49, no. 12, pp. 1605-1615, Dec. 2011.

[24] Y. M. Mo, J. Zhang, B. H. Qin, and J. C. Lv, “Application of a Method on Optimization of Thin-Walled Column's Crashworthiness Based on Metamodel," Appl. Mech. Mater., vol. 526, pp. 109-114, Feb. 2014.

[25] E. Acar, M. A. Guler, B. Gerçeker, M. E. Cerit, and B. Bayram, "Multi-objective crashworthiness optimization of tapered thin-walled tubes with axisymmetric indentations," Thin-Walled Struct., vol. 49, no. 1, pp. 94-105, Jan. 2011

[26] G. Sun, F. Xu, G. Li, and Q. Li, "Crashing analysis and multiobjective optimization for thin-walled structures with functionally graded thickness," Int. J. Impact Eng. , vol. 64, pp. 6274,2014

[27] H. Yin, G. Wen, H. Fang, Q. Qing, X. Kong, J. Xiao, and Z. Liu, "Multiobjective crashworthiness optimization design of functionally graded foam-filled tapered tube based on dynamic ensemble metamodel," Mater. Des., vol. 55, pp. 747-757, Mar. 2014.

[28] X. Song, G. Sun, G. Li, W. Gao, and Q. Li, "Crashworthiness optimization of foam-filled tapered thin-walled structure using multiple surrogate models," Struct. Multidiscip. Optim., vol. 47, no. 2, pp. 221-231, 2012.

[29] H. Yin, G. Wen, Z. Liu, and Q. Qing, "Crashworthiness optimization design for foam-filled multi-cell thin-walled structures," Thin-Walled Struct., vol. 75, pp. 8-17, Feb. 2014.

[30] J. Bi, H. Fang, Q. Wang, and X. Ren, "Modeling and optimization of foam-filled thin-walled columns for crashworthiness designs," Finite Elem. Anal. Des. , vol. 46, no. 9, pp. 698-709, 2010. 
[31] Z. Zhang, S. Liu, and Z. Tang, "Comparisons of honeycomb sandwich and foam-filled cylindrical columns under axial crushing loads," Thin-Walled Struct., vol. 49, no. 9, pp. 10711079, 2011.

[32] J. Paz, J. Díaz, L. Romera, and M. Costas, "Size and shape optimization of aluminum tubes with GFRP honeycomb reinforcements for crashworthy aircraft structures," Compos. Struct., vol. 133, pp. 499-507, Dec. 2015.

[33] Z. Ahmad and D. P. Thambiratnam, "Crushing response of foam-filled conical tubes under quasi-static axial loading," Mater. Des., vol. 30, no. 7, pp. 2393-2403, Aug. 2009.

[34] H. Yin, H. Fang, Y. Xiao, G. Wen, and Q. Qing, "Multiobjective robust optimization of foam-filled tapered multi-cell thinwalled structures," Struct. Multidiscip. Optim., Jul. 2015.

[35] Z. Ahmad and D. P. Thambiratnam, "Dynamic computer simulation and energy absorption of foam-filled conical tubes under axial impact loading," Comput. Struct., vol. 87, no. 3-4, pp. 186-197, Feb. 2009.

[36] J. J. Carruthers, A. P. Kettle, and A. M. Robinson, "Energy Absorption Capability and Crashworthiness of Composite Material Structures: A Review," Appl. Mech. Rev., vol. 51, no. 10, p. 635, Oct. 1998.

[37] L. Lanzi, L. M. . Castelletti, and M. Anghileri, "Multiobjective optimisation of composite absorber shape under crashworthiness requirements," Compos. Struct., vol. 65, no. 3-4, pp. 433-441, Sep. 2004.

[38] L. Cui, S. Kiernan, and M. D. Gilchrist, "Designing the energy absorption capacity of functionally graded foam materials," Mater. Sci. Eng. A, vol. 507, no. 1-2, pp. 215-225, May 2009.

[39] J. Zhang, P. Supernak, S. Mueller-Alander, and C. H. Wang, "Improving the bending strength and energy absorption of corrugated sandwich composite structure," Mater. Des., vol. 52, pp. 767-773, Dec. 2013.

[40] S. Duan, Y. Tao, X. Han, X. Yang, S. Hou, and Z. Hu, "Investigation on structure optimization of crashworthiness of fiber reinforced polymers materials," Compos. Part B Eng., vol. 60, pp. 471-478, Apr. 2014.

[41] S. Shinde, P. Bandi, D. Detwiler, and A. Tovar, "Structural Optimization of Thin-Walled Tubular Structures for Progressive Buckling Using Compliant Mechanism Approach," SAE Int. J. Passeng. Cars - Mech. Syst., vol. 6, no. 1, pp. 109-120, 2013.

[42] K. Liu and A. Tovar, "An efficient 3D topology optimization code written in Matlab," Struct. Multidiscip. Optim., vol. 50, no. 6, pp. 1175-1196, Jun. 2014.

[43] J. B. MacQueen, "Some Methods for classification and Analysis of Multivariate Observations," 5-th Berkeley Symposium on Mathematical Statistics and Probability, vol. 1. University of California Press, pp. 281-297, 1967.

[44] D. MacKay, Information Theory, Inference, and Learning Algorithms. Cambridge University Press, 2003.
[45] J. Song and H. Wang, "Ckmeans.1d.dp: Optimal k-means clustering for one-dimensional data." 2014.

[46] H. Wang and M. Z. Song, "Ckmeans.1d.dp: optimal kmeans clustering in one dimension by dynamic programming," $R J$, vol. 3, no. 2, pp. 29-33, 2011.

[47] B. Hassani, "Homogenization and Topological Structural Optimization," University of Wales, Swansea, 1996.

[48] Z. Hashin and S. Shtrikman, "A variational approach to the theory of the elastic behaviour of multiphase materials," J. Mech. Phys. Solids, vol. 11, no. 2, pp. 127-140, 1963.

[49] D. R. JONES, M. SCHONLAU, and W. J. WELCH, "Efficient Global Optimization of Expensive Black-Box Functions," J. Glob. Optim., vol. 13, pp. 455-492, 1998. 